\title{
On a Class of Function of Bounded Turning with Negative Coefficients Associated with a Generalized Multiplier Transformation
}

\author{
M. O. OLUWAYEMI ${ }^{1,2}$, B. O. MOSES ${ }^{3}$, J. O. HAMZA ${ }^{4}$ \\ ${ }^{1}$ Landmark University SDG 4 (Quality Education Research Group), Omu-Aran, NIGERIA \\ ${ }^{2}$ Department of Mathematics, Landmark University, P.M.B. 1001, Omu-Aran, NIGERIA \\ ${ }^{3}$ Department of Mathematics, American University of Nigeria, Yola, NIGERIA ${ }^{4}$ Department of \\ Mathematics, University of Lagos, Akoka, NIGERIA 1,2
}

\begin{abstract}
The study of univalent functions and its applications is an hallmark of geometric function theory. Since univalent functions are analytic and has one-to-one mapping, it has a wide range of applications in the fields of studies where transformations (enlargements and reductions) are done. The functions also have angle and orientation preserving properties among other uses. Many authors have defined and studied various classes of univalent functions using different approaches and tools. In this study however, the authors used a generalized multiplier transformation to define a and investigate a new class of functions $T_{\sigma}^{n}(\lambda)$. Various properties of the class of functions were investigated. The results extend some known results in literature.
\end{abstract}

Key-Words: - Univalent, unit disc, multiplier transformation, function of bounded turning, starlike functions, convex functions, and coefficients

Received: April 24, 2021. Revised: January 10, 2022. Accepted: January 27, 2022. Published: February 25, 2022.

\section{Introduction and Preliminaries}

Let

$$
f(z)=z+\sum_{k=2}^{\infty} a_{k} z^{k}
$$

which are analytic in the open unit disk $\mathbb{U}=\{z \in$ $\mathbb{C}:|z|<1\}$ and normalised by $f(0)=0$ and $f^{\prime}(0)=1$.

We denote by $T$ the subclass of $\mathrm{S}$ which are normalized univalent function of the form

$$
f(z)=z-\sum_{k=2}^{\infty} a_{k} z^{k}, a_{k}>0
$$

that are analytic and univalent in the open unit disk $\mathbb{U}$. The function of the form (2) was first introduced in [13]. Functions of negative coefficients were also investigated in [2], [5], [6], [8], [9] and [10]. Univalent functions have applications in many areas of science and engineering, The simplest example of a univalent function is the identity function $e(z)=$ z. Other examples are the circular function $\mathrm{f}(\mathrm{z})=$ $\operatorname{sinz}$ which is univalent in the disc $|z|<\frac{\pi}{2}$ and the Koebe function

\subsection{Multiplier Transformations (Differential Operator)}

Since the introduction of differentiatial operator as multiplier transformation in the geometric function theories, various authors such as [1], [4], [11], [12], [14] and [15] have continued to extend the transformation and used as a tool to define, study and establish properties of the class of functions.
Let

$$
\begin{aligned}
& D_{\mu, \lambda, \eta}^{n}(\sigma, \xi, \omega, \epsilon) f(z)=z-\sum_{k=2}^{\infty}(1+ \\
& \left.\frac{(k-1)\left[\lambda(\omega-\xi)^{\sigma}+k(\eta+\epsilon)\right]}{\mu+\omega}\right)^{n} a_{k} z^{k} .
\end{aligned}
$$

For convenience, we let

$f(z)=z-\sum_{k=2}^{\infty} \Phi a_{k} z^{k}$.

So that

$$
\Phi=\left(1+\frac{(k-1)\left[\lambda(\omega-\xi)^{\sigma}+k(\eta+\epsilon)\right]}{\mu+\omega}\right)^{n} .
$$

The function $f(z)$ defined in (4) is associated with the multiplier transformation $D_{\mu, \lambda, \eta}^{n}(\sigma, \xi, \omega, \epsilon)$ defined in [8] where $\mu, \lambda, \eta, \sigma, \xi, \epsilon \geq 0$ with $\omega>0$ such that $\xi<\omega$ and $n \in \mathbb{N}_{0}=\mathbb{N} \cup\{0\}$. The operator generalises the operators defined in [4], [12], [14], and [15]. The operator also reduces to (2) when $n=0$.

\subsection{Class $T_{\sigma}^{n}(\lambda)$}

Definition 1.1 A function $f(z)$ defined by (2) is said to belong to class $T_{\sigma}^{n}(\lambda)$ if $D_{\mu, \lambda, \eta}^{n}(\sigma, \xi, \omega, \epsilon) f(z)$ is a function of bounded turning. That is, if

$\left(D_{\mu, \lambda, \eta}^{n}(\sigma, \xi, \omega, \epsilon) f(z)\right)^{\prime}>0$.

Investigation of classes of univalent functions is a great deal in geometric functions theory (GFT). Various authors have investigated different forms of functions such as starlike functions, convex functions $K$, close to convex functions $K \mathcal{L}$ and 
functions of bounded turning $B$ among others. Ibrahim and Kılıçman in 16] considered a relationship between $B, K$ and $K \mathcal{L}$ as classes of univalent functions. Deng in [3] studied some geometric properties of a univalent functions with negative coefficients which was first defined in [13] while Fadipe-Joseph and Oluwayemi in [7] defined and investigated class $C_{\alpha}$ as a class of convex univalent functions. Motivated by the work of [3] and [8], the following results were presented using a differential operator. Other approaches such as subordination principle and convolution among others could also be used in investigating a class of function.

\section{Main Results}

Theorem 2.1 A function $f(z)$ defined by (4) belongs to class $T_{\sigma}^{n}(\lambda)$ if and only if

$\sum_{k=2}^{\infty} k\left(1+\frac{(k-1)\left[\lambda(\omega-\xi)^{\sigma}+k(\eta+\epsilon)\right]}{\mu+\omega}\right)^{n}\left|a_{k}\right| \leq 1$.

\section{Proof:}

It's necessary to show that $\sum_{k=2}^{\infty} k(1+$ $\left.\frac{(k-1)\left[\lambda(\omega-\xi)^{\sigma}+k(\eta+\epsilon)\right]}{\mu+\omega}\right)^{n} \leq 1 \quad$ if $\quad f(z) \in T_{\sigma}^{n}(\lambda)$. Suppose $f(z) \in T_{\sigma}^{n}(\lambda)$, then $f(z)$ is univalent in $\mathrm{U}$. Consequently,

$$
\begin{aligned}
& f^{\prime}(z) \\
& =1 \\
& -\sum_{k=2}^{\infty} k(1 \\
& \left.+\frac{(k-1)\left[\lambda(\omega-\xi)^{\sigma}+k(\eta+\epsilon)\right]}{\mu+\omega}\right)^{n}\left|a_{k}\right| z^{k-1}
\end{aligned}
$$

is non negative which implies that $f^{\prime}(z) \neq 0$. Thus,

$$
\begin{aligned}
& f^{\prime}(r) \\
& =1 \\
& -\sum_{k=2}^{\infty} k(1 \\
& \left.+\frac{(k-1)\left[\lambda(\omega-\xi)^{\sigma}+k(\eta+\epsilon)\right]}{\mu+\omega}\right)^{n}\left|a_{k}\right| r^{k-1} \\
& \neq 0 ; \quad(|z|=r) .
\end{aligned}
$$

Assume

$$
\sum_{k=2}^{\infty} k\left(1+\frac{(k-1)\left[\lambda(\omega-\xi)^{\sigma}+k(\eta+\epsilon)\right]}{\mu+\omega}\right)^{n}\left|a_{k}\right|
$$

then there exists $n \in \mathbb{N}_{0}$ such that

$$
\sum_{k=2}^{\infty} k\left(1+\frac{(k-1)\left[\lambda(\omega-\xi)^{\sigma}+k(\eta+\epsilon)\right]}{\mu+\omega}\right)^{n}\left|a_{k}\right|
$$

Hence, we have $r_{0} \in(0,1)$ such that $\sum_{k=2}^{\infty} k(1$

$\left.+\frac{(k-1)\left[\lambda(\omega-\xi)^{\sigma}+k(\eta+\epsilon)\right]}{\mu+\omega}\right)^{n}\left|a_{k}\right| r_{0}^{k-1}>1$.

Thus,

$f^{\prime}\left(r_{0}\right)$

$=1$

$-\sum_{k=2}^{\infty} k(1$

$\left.+\frac{(k-1)\left[\lambda(\omega-\xi)^{\sigma}+k(\eta+\epsilon)\right]}{\mu+\omega}\right)^{n}\left|a_{k}\right| r_{0}^{k-1}$

$-\sum_{k=2}^{\infty n} k(1$

$\left.+\frac{(k-1)\left[\lambda(\omega-\xi)^{\sigma}+k(\eta+\epsilon)\right]}{\mu+\omega}\right)^{n}\left|a_{k}\right| r_{0}^{k-1}<0$.

But $f^{\prime}(r)$ is continuous and $f^{\prime}(0)=1$. Hence, we can find $r_{1} \in\left(0, r_{0}\right)$ such that $f^{\prime}\left(r_{1}\right)=0$ by intermediate value theorem which contradicts the assumption. Hence,

$\sum_{k=2}^{\infty} k\left(1+\frac{(k-1)\left[\lambda(\omega-\xi)^{\sigma}+k(\eta+\epsilon)\right]}{\mu+\omega}\right)^{n} \leq 1$

as required.

Conversely, assume

$\sum_{k=2}^{\infty} k\left(1+\frac{(k-1)\left[\lambda(\omega-\xi)^{\sigma}+k(\eta+\epsilon)\right]}{\mu+\omega}\right)^{n} \leq 1$.

Then

$$
=\sum_{k=2}^{\operatorname{Ref}_{\infty}^{\prime}(z)} k\left(1+\frac{(k-1)\left[\lambda(\omega-\xi)^{\sigma}+k(\eta+\epsilon)\right]}{\mu+\omega}\right)^{n}
$$$$
>1-\sum_{k=2}^{\infty} k\left(1+\frac{(k-1)\left[\lambda(\omega-\xi)^{\sigma}+k(\eta+\epsilon)\right]}{\mu+\omega}\right)^{n} \geq 0 .
$$

Thus,

$$
\frac{1}{2}=\int_{0}^{1} \operatorname{Re} f^{\prime}(z)
$$

The result is sharp for

$$
\begin{aligned}
& f(z)=z-\sum_{k=2}^{\infty}(1+ \\
& \left.\frac{(k-1)\left[\lambda(\omega-\xi)^{\sigma}+k(\eta+\epsilon)\right]}{\mu+\omega}\right)^{n} a_{k} z^{k} .
\end{aligned}
$$

Corollary 2.1 A function $f(z)$ defined by (4) belongs to the class $T_{0}^{n}(\lambda)$ if and only if

$\sum_{k=2}^{\infty} k\left(1+\frac{[\lambda(k-1)+k(k-1)(\eta+\epsilon)]}{\mu+\omega}\right)^{n}\left|a_{k}\right| \leq 1$.

Corollary 2.2 A function $f(z)$ defined by (4) belongs to the class $T_{0}^{n}(0)$ if and only if

$\sum_{k=2}^{\infty} k\left(1+\frac{[k(k-1)(\eta+\epsilon)]}{\mu+\omega}\right)^{n}\left|a_{k}\right| \leq 1$.

Corollary 2.3 A function $f(z)$ defined by (4) belongs to the class $T_{\sigma}^{0}(\lambda)$ if and only if

$$
\sum_{k=2}^{\infty} k\left|a_{k}\right| \leq 1 .
$$


Remark 2.1 The class $T_{\sigma}^{0}(\lambda) \equiv T$ investigated in [3].

Theorem 2.2 The class $T_{\sigma}^{n}(\lambda)$ is convex.

\section{Proof:}

Let $f \in T_{\sigma}^{n}(\lambda)$ be defined as (4). Then, there exists a function

$f_{i}(z)=z-\sum_{k=2}^{\infty} \Phi a_{k, i} z^{k}, i=1,2 \quad z \in U$.

in $T_{\sigma}^{n}(\lambda)$ such that

$g(z)=z-\sum_{k=2}^{\infty}\left(\varsigma_{1} \Phi a_{k, 1}+\varsigma_{2} \Phi a_{k, 2}\right) z^{k} \quad z \in U$

where $\varsigma_{1}$ and $\varsigma_{2}$ are non-negative and $\varsigma_{1}=1-\varsigma_{2}$.

Then,

$$
[g(z)]^{\prime}=1-\sum_{k=2}^{\infty} k\left(\varsigma_{1} \Phi a_{k, 1}+\varsigma_{2} \Phi a_{k, 2}\right) z^{k-1}
$$

and

$\operatorname{Re}[g(z)]^{\prime}=\operatorname{Re}\left[1-\sum_{k=2}^{\infty} k\left(\varsigma_{1} \Phi a_{k, 1}+\right.\right.$

$\left.\left.\varsigma_{2} \Phi a_{k, 2}\right) z^{k-1}\right]$

$=1-\operatorname{Re}\left[\varsigma_{i} \sum_{k=2}^{\infty} k \Phi a_{k, 1} z^{k-1}\right]+$

$\operatorname{Re}\left[\varsigma_{2} \sum_{k=2}^{\infty} k \Phi a_{k, 1} z^{k-1}\right]$.

Since $f_{1}, f_{2} \in T_{\sigma}^{n}(\lambda)$. Then,

$\operatorname{Re}\left[\varsigma_{i} \sum_{k=2}^{\infty} k \Phi a_{k, 1} z^{k-1}\right]>\varsigma_{i}(0-1) ; \quad(i=1,2)$

Using (15) in (14),

$$
\begin{gathered}
\operatorname{Re}[g(z)]^{\prime}>1+\varsigma_{1}(0-1)+\varsigma_{2}(0-1) \\
=1-\left(\varsigma_{1}+\varsigma_{2}\right) .
\end{gathered}
$$

Since $\varsigma_{1}+\varsigma_{2}=1$, it follows that

Consequently

$$
\operatorname{Re}[g(z)]^{\prime}>0 \text {. }
$$

$$
g(z)=\varsigma_{1} f_{1}(z)+\varsigma_{2} f_{2}(z)
$$

which implies that the class $T_{\sigma}^{n}(\lambda)$ is convex.

Theorem 2.3 Let $f(z) \in T_{\sigma}^{n}(\lambda)$. Then,

$$
\begin{aligned}
r-\frac{1}{2}\left(1+\frac{\left[\lambda(\omega-\xi)^{\sigma}+2(\eta+\epsilon)\right]}{\mu+\omega}\right)^{n} r^{2} \leq|f(z)| \\
\leq r \\
+\frac{1}{2}(1 \\
\left.+\frac{\left[\lambda(\omega-\xi)^{\sigma}+2(\eta+\epsilon)\right]}{\mu+\omega}\right)^{n} r^{2}
\end{aligned}
$$

with equality for

$$
\begin{aligned}
& f(z) \\
& =z-\frac{1}{2}\left(1+\frac{\left[\lambda(\omega-\xi)^{\sigma}+2(\eta+\epsilon)\right]}{\mu+\omega}\right)^{n} z^{2} ;|z| \\
& =r .
\end{aligned}
$$

\section{Proof:}

Let $T_{\sigma}^{n}(\lambda)$, then we have that (8) holds. Also,

$$
\begin{aligned}
& 2 \sum_{k=2}^{\infty}\left(1+\frac{(k-1)\left[\lambda(\omega-\xi)^{\sigma}+k(\eta+\epsilon)\right]}{\mu+\omega}\right)^{n}\left|a_{k}\right| \\
& \leq \sum_{k=2}^{\infty} k(1 \\
& \left.+\frac{(k-1)\left[\lambda(\omega-\xi)^{\sigma}+k(\eta+\epsilon)\right]}{\mu+\omega}\right)^{n}\left|a_{k}\right| \leq 1
\end{aligned}
$$

also from Theorem 2.1. Hence,

$$
\begin{aligned}
& |f(z)| \\
& \leq r \\
& +\sum_{k=2}^{\infty}(1 \\
& \left.+\frac{(k-1)\left[\lambda(\omega-\xi)^{\sigma}+k(\eta+\epsilon)\right]}{\mu+\omega}\right)^{n}\left|a_{k}\right| r^{k} \\
& \leq r \\
& +r^{2} \sum_{k=2}^{\infty}(1 \\
& \left.+\frac{(k-1)\left[\lambda(\omega-\xi)^{\sigma}+k(\eta+\epsilon)\right]}{\mu+\omega}\right)^{n}\left|a_{k}\right| \\
& \leq r+\frac{1}{2}\left(1+\frac{\left[\lambda(\omega-\xi)^{\sigma}+2(\eta+\epsilon)\right]}{\mu+\omega}\right)^{n} r^{2}
\end{aligned}
$$

and

$$
\begin{aligned}
& |f(z)| \\
& \geq r \\
& -\sum_{k=2}^{\infty}(1 \\
& \left.+\frac{(k-1)\left[\lambda(\omega-\xi)^{\sigma}+k(\eta+\epsilon)\right]}{\mu+\omega}\right)^{n}\left|a_{k}\right| r^{k} \\
& \geq r \quad \\
& +r^{2} \sum_{k=2}^{\infty}(1 \\
& \left.+\frac{(k-1)\left[\lambda(\omega-\xi)^{\sigma}+k(\eta+\epsilon)\right]}{\mu+\omega}\right)^{n}\left|a_{k}\right| \\
& \geq r+\frac{1}{2}\left(1+\frac{\left[\lambda(\omega-\xi)^{\sigma}+2(\eta+\epsilon)\right]}{\mu+\omega}\right)^{n} r^{2} .
\end{aligned}
$$

The results follows from the above expressions.

Corollary 2.4 Let $f(z) \in T_{\sigma}^{0}(\lambda)$. Then,

with equality for

$$
r-\frac{1}{2} r^{2} \leq|f(z)| \leq r+\frac{1}{2} r^{2}
$$

$$
f(z)=z-\frac{1}{2} z^{2} ;|z|=r .
$$

Theorem 2.4 Let $f(z) \in T_{\sigma}^{n}(\lambda)$. The disk $|z|$ is mapped onto a domaiin that contains the disk

with equality for

$$
|\omega| \leq \frac{1}{2}
$$


$f(z)$

$$
\begin{aligned}
& =z-\frac{1}{2}\left(1+\frac{\left[\lambda(\omega-\xi)^{\sigma}+2(\eta+\epsilon)\right]}{\mu+\omega}\right)^{n} z^{2} ;|z| \\
& =r .
\end{aligned}
$$

\section{Proof:}

Suppose $f(z) \in T_{\sigma}^{n}(\lambda)$, we have from Theorem 2.3 that

$$
|f(z)| \leq r+\frac{1}{2}\left(1+\frac{\left[\lambda(\omega-\xi)^{\sigma}+2(\eta+\epsilon)\right]}{\mu+\omega}\right)^{n} r^{2} .
$$

The result follows as $r \rightarrow 1$.

Theorem 2.5 Let $f(z) \in T_{\sigma}^{n}(\lambda)$. Then,

$$
\begin{aligned}
1-\left(1+\frac{\left[\lambda(\omega-\xi)^{\sigma}+2(\eta+\epsilon)\right]}{\mu+\omega}\right)^{n} r \leq|f(z)| \\
\leq 1 \\
+(1 \\
\left.+\frac{\left[\lambda(\omega-\xi)^{\sigma}+2(\eta+\epsilon)\right]}{\mu+\omega}\right)^{n} r
\end{aligned}
$$

with equality for

$$
\begin{aligned}
& f(z) \\
& =z-\frac{1}{2}\left(1+\frac{\left[\lambda(\omega-\xi)^{\sigma}+2(\eta+\epsilon)\right]}{\mu+\omega}\right)^{n} z^{2} ;|z| \\
& =r .
\end{aligned}
$$

\section{Proof:}

Suppose $f(z) \in T_{\sigma}^{n}(\lambda)$. Then,

$$
\begin{aligned}
& |f(z)| \\
& \leq 1 \\
& +\sum_{k=2}^{\infty} k(1 \\
& \left.+\frac{(k-1)\left[\lambda(\omega-\xi)^{\sigma}+k(\eta+\epsilon)\right]}{\mu+\omega}\right)^{n}\left|a_{k}\right| r^{k-1}
\end{aligned}
$$

$\leq 1$

$+r \sum_{k=2}^{\infty} k(1$

$\left.+\frac{(k-1)\left[\lambda(\omega-\xi)^{\sigma}+k(\eta+\epsilon)\right]}{\mu+\omega}\right)^{n}\left|a_{k}\right| \leq 1+r$

and

$|f(z)|$

$\geq 1$

$$
\begin{aligned}
& -\sum_{k=2}^{\infty} k(1 \\
& \left.+\frac{(k-1)\left[\lambda(\omega-\xi)^{\sigma}+k(\eta+\epsilon)\right]}{\mu+\omega}\right)^{n}\left|a_{k}\right| r^{k-1}
\end{aligned}
$$

$\geq 1$

$$
\begin{aligned}
& -r \sum_{k=2}^{\infty} k(1 \\
& \left.+\frac{(k-1)\left[\lambda(\omega-\xi)^{\sigma}+k(\eta+\epsilon)\right]}{\mu+\omega}\right)^{n}\left|a_{k}\right| \geq 1-r
\end{aligned}
$$

as required.

Corollary 2.5 Let $f(z) \in T_{\sigma}^{0}(\lambda)$. Then,

$$
1-r \leq|f(z)| \leq 1+r
$$

with equality for

$$
f(z)=z-\frac{1}{2} z^{2} \quad|z|=r .
$$

Let $j \in \mathbb{N}$ such that $j=1, \ldots l$. We define the functions

$$
\begin{aligned}
& f_{j}(z)=z-\sum_{k=2}^{\infty}(1+ \\
& \left.\frac{(k-1)\left[\lambda(\omega-\xi)^{\sigma}+k(\eta+\epsilon)\right]}{\mu+\omega}\right)^{n} a_{k, j} z^{k}, a_{k, j} \geq 0 .
\end{aligned}
$$

Theorem 2.6 Let the functions $f_{j}(z)$ defined by $(16$ be in the class $T_{\sigma}^{n}(\lambda)$. Then, the function $G(z)$ defined by

$G(z)=z-\sum_{k=2}^{\infty} p_{k} z^{k}, p_{k} \geq 0$.

Then, $G(z) \in T_{\sigma}^{n}(\lambda)$ where

$p_{k}=\frac{1}{l} \sum_{k=2}^{l} a_{k, j}$

\section{Proof:}

Let $f_{j}(z) \in T_{\sigma}^{n}(\lambda)$. Then using (16) and by Theorem 2.1,

$$
\begin{aligned}
& \sum_{k=2}^{\infty} k\left(1+\frac{(k-1)\left[\lambda(\omega-\xi)^{\sigma}+k(\eta+\epsilon)\right]}{\mu+\omega}\right)^{n} a_{k, j} \\
& \in \mathbb{N}, \quad(j=1 \ldots l) .
\end{aligned}
$$

Hence,

$$
\begin{gathered}
\sum_{k=2}^{\infty}\left(1+\frac{(k-1)\left[\lambda(\omega-\xi)^{\sigma}+k(\eta+\epsilon)\right]}{\mu+\omega}\right)^{n} p_{k} \\
=\sum_{k=2}^{\infty}(1 \\
\left.+\frac{(k-1)\left[\lambda(\omega-\xi)^{\sigma}+k(\eta+\epsilon)\right]}{\mu+\omega}\right)^{n}\left(\frac{1}{l} \sum_{k=2}^{l} a_{k, j}\right) \\
=\frac{1}{l} \sum_{k=2}^{l}\left\{\sum_{k=2}^{\infty}(1\right. \\
\left.\left.+\frac{(k-1)\left[\lambda(\omega-\xi)^{\sigma}+k(\eta+\epsilon)\right]}{\mu+\omega}\right)^{n} a_{k, j}\right\} \\
\leq \frac{1}{l} \sum_{k=2}^{l} a_{k, j}=1 .
\end{gathered}
$$

Theorem 2.7 Suppose a function $f(z)$ defined by (4) belongs to class $T_{\sigma}^{n}(\lambda)$ and there exists $\gamma \in \mathbb{R}$ such that $\gamma>-1$. Then, the function $h(z)$ defined by

$$
h(z)=\frac{\gamma+1}{z^{\gamma}} \int_{0}^{z} x^{\gamma-1} f(x) d x
$$

also belongs to the class $T_{\sigma}^{n}(\lambda)$.

\section{Proof:}

Let $f(z) \in T_{\sigma}^{n}(\lambda)$. Then, we define 
$h(z)$

$=z$

$-\sum_{k=2}^{\infty}(1$

$\left.+\frac{(k-1)\left[\lambda(\omega-\xi)^{\sigma}+k(\eta+\epsilon)\right]}{\mu+\omega}\right)^{n}\left|b_{k}\right| z^{k}$

where

$$
\left|b_{k}\right|=\frac{\gamma+1}{\gamma+k}\left|a_{k}\right|
$$

So that

$\sum_{k=2}^{\infty} k\left(1+\frac{(k-1)\left[\lambda(\omega-\xi)^{\sigma}+k(\eta+\epsilon)\right]}{\mu+\omega}\right)^{n}\left|b_{k}\right|$

$=\sum_{k=2}^{\infty} k\left(1 \frac{(k-1)\left[\lambda(\omega-\xi)^{\sigma}+k(\eta+\epsilon)\right]}{\mu+\omega}\right)^{n} \frac{\gamma+1}{\gamma+k}\left|a_{k}\right|$

$\sum_{k=2}^{\infty} k\left(1+\frac{(k-1)\left[\lambda(\omega-\xi)^{\sigma}+k(\eta+\epsilon)\right]}{\mu+\omega}\right)^{n}\left|a_{k}\right|$

$\leq 1$.

The result follows from Theorem 2.1.

Theorem 2.8 Let $\gamma \in \mathbb{R}$ such that $\gamma>-1$. If $h(z) \in T_{\sigma}^{n}(\lambda)$, then the function $h(z)$ defined by (18) is univalent in $|z|<R$

where

$R=i n f_{k}[(1+$

$\left.\left.\frac{(k-1)\left[\lambda(\omega-\xi)^{\sigma}+k(\eta+\epsilon)\right]}{\mu+\omega}\right)^{n}\left(\frac{\gamma+1}{\gamma+k}\right)\right]^{\frac{1}{k-1}}, \quad k \geq 2$.

\section{Proof:}

Let

$$
\begin{aligned}
& h(z) \\
& =z \\
& -\sum_{k=2}^{\infty}(1 \\
& \left.+\frac{(k-1)\left[\lambda(\omega-\xi)^{\sigma}+k(\eta+\epsilon)\right]}{\mu+\omega}\right)^{n}\left|a_{k}\right| z^{k} .
\end{aligned}
$$

Then from (18),

We now need to show that $|h(z)-1|<1$ in $|z|<$ $R$. But

$\left|h^{\prime}(z)-1\right|$

$\leq \sum_{k=2}^{\infty}(1$

$\left.+\frac{(k-1)\left[\lambda(\omega-\xi)^{\sigma}+k(\eta+\epsilon)\right]}{\mu+\omega}\right)^{n}\left(\frac{\gamma+1}{\gamma+1}\right)\left|a_{k}\right||z|^{k-1}$.

We have that since $h(z) \in T_{\sigma}^{n}(\lambda)$. Then,

$\sum_{k=2}^{\infty}(1+$

$\left.\frac{(k-1)\left[\lambda(\omega-\xi)^{\sigma}+k(\eta+\epsilon)\right]}{\mu+\omega}\right)^{n}\left(\frac{\gamma+1}{\gamma+1}\right)\left|a_{k}\right||z|^{k-1}<1$ implies that $\left|h^{\prime}(z)-1\right|<1$. Using

$\sum_{k=2}^{\infty} k\left(1+\frac{(k-1)\left[\lambda(\omega-\xi)^{\sigma}+k(\eta+\epsilon)\right]}{\mu+\omega}\right)^{n}\left|a_{k}\right|$

(20) will hold true if

$k(1$

$\left.+\frac{(k-1)\left[\lambda(\omega-\xi)^{\sigma}+k(\eta+\epsilon)\right]}{\mu+\omega}\right)^{n} \frac{\gamma+1}{\gamma+k}|z|^{k-1}$

$\leq k$

which implies that

$$
\begin{aligned}
& \leq[(1 \\
& \left.\left.+\frac{(k-1)\left[\lambda(\omega-\xi)^{\sigma}+k(\eta+\epsilon)\right]}{\mu+\omega}\right)^{n}\left(\frac{\gamma+1}{\gamma+k}\right)\right]^{\frac{1}{k-1}} \\
& \leq 1
\end{aligned}
$$

which completes the proof.

The result is sharp for

$$
\begin{aligned}
& h(z) \\
& =z \\
& -[(1 \\
& \left.\left.+\frac{(k-1)\left[\lambda(\omega-\xi)^{\sigma}+k(\eta+\epsilon)\right]}{\mu+\omega}\right)^{n}\left(\frac{\gamma+1}{\gamma+k}\right)\right] z^{k}
\end{aligned}
$$

Corollary 2.6 Let $\gamma \in \mathbb{R}$ such that $\gamma>-1$. If $h(z) \in T_{\sigma}^{0}(\lambda)$, then the function $h(z)$ defined by (18) is univalent in $|z|<R$

where

$R=i n f_{k}\left(\frac{\gamma+1}{\gamma+1}\right)^{\frac{1}{k-1}}, \quad k \geq 2$.

\section{Conclusion}

The result extend some known results in literature. In particular, the class of function defined in [3] is extended in the present study. The results in the study are generalised in this paper. The class of functions studied in the work find applications in the areas where mappings are done.

\section{Acknowledgement:}

The authors acknowledge their respective institution.

\section{References:}

[1] Al-Oboudi F. M. (2004). On univalent functions defined by a generalized Sălăgean operator, International Journal of Mathematics and Mathematical Science, 27, 1419-1436. 
[2] Aouf, M. K. and Cho, Nak Eun (1998). On a certain subclass of analytic functions with negative coefficients, Tr. J. of Mathematics, TÜBITAK, 22, 15-32.

[3] Deng Q. (2007). On univalent functions with negative coefficients, Applied Mathematics and Computation, 1675-1682.

[4] Catas A. (2007). On certain class of p-valent functions defined by new multiplier transformations, Proceedings book of the international symposium on geometric function theory and applications, 20-24, 2007, TC Isambul Kultur University, Turkey, 241250.

[5] Ezhilarasi R., Sudharsan T.V., Sivasubramanian S. (2020). On certain subclass ofunivalent functions with finitely many fixed coefficients defined by Bessel function, Malaya Journal of Matematik, 8(3), 1085-1091.

[6] Ezhilarasi R., Sudharsan T. V., Mohd M. H., Subramanian K. G. (2017). Connections between Certain Subclasses of Analytic Univalent Functions Based on Operators, Journal of Complex Analysis, 1-5.

[7] Fadipe-Joseph O. A., Oluwayemi M. O. (2021). Sufficient conditions for class $C_{\alpha}$, International Journal of Mathematics and Computer Science (IJMCS), 16(2), 154-159.

[8] Oluwayemi M. O., Fadipe-Joseph O. A., (2022). A New Class of Function with Finitely many Fixed Points, Abstract and Applied Analysis, DOI: $\quad 10.1155 / 2022 / 9936129$ https://www.hindawi.com/journals/aaa/conten $\underline{\text { ts }}$

/[9] Oluwayemi M. O., Okoro J. O. (2021). Certain results on a class of functions with negative coefficients International Journal of Mathematics and Computer Science, 16(4), $1295-1302$.

[10] Oluwayemi M. O., Faisal I. (2022), A new family of analytic functions associated with multiplier transformation, Scientific Africa, 12, $1-9$ https://doi.org/10.1016/j.sciaf.2021.e00754.

[11] Ruscheweyh, S (1975). New criteria for univalent functions, Proceeding of American Mathematical Society 49, 109-115.

[12] Sălăgean, G.S. (1983). Subclasses of univalent functions, Complex Analysis-Fifth RomanianFinnish Seminar, Springer Berlin Heidelberg, 362-372.

[13] Silverman H. (1975). Univalent functions with negative coefficients, Proceeding American Mathematical Society, 51, 109-116.
[14] Swamy S. R. (2012). On univalent functions defined by a new generalized multiplier differential operator, Journal of Mathematical and Computational Science, 2(2012), 1233-1240

[15] Uralegaddi B. A. , Somanatha C. (1992). Certain classes of univalent functions, In: Current Topics in Analytic Function Theory, Eds. Srivastava, H. M., Owa, S., World Scientific Publishing Company, Singapore, 371-374.

[16] Ibrahim R. W., Kılıçman A. (2012). A note on the class of functions with bounded turning, Abstract and Applied Analysis, vol. 2012, doi.10.1155/2012/820696

\section{Contribution of Individual Authors to the Creation of a Scientific Article (Ghostwriting Policy)}

Oluwayemi M.O. did conceptualization, the initial investigation of the work and also prepared the original draft, Hamzat J. O. reviewed the work after the initial draft to ascertain the correctness of the work and while Moses B. O. worked on the methodology and validation of the study. All authors agreed upon the final work and approved the final draft accordingly.

\section{Sources of Funding for Research Presented in a Scientific Article or Scientific Article Itself}

The authors report that they do not have any form of financial support for the work.

\section{Creative Commons Attribution License 4.0 (Attribution 4.0 International, CC BY 4.0)}

This article is published under the terms of the Creative Commons Attribution License 4.0 https://creativecommons.org/licenses/by/4.0/deed.en _US 